\title{
Sum Frequency Generation Vibrational Spectroscopy of Pyridine Hydrogenation on Platinum Nanoparticles
}

\author{
Kaitlin M. Bratlie, ${ }^{1,2, \ddagger}$ Kyriakos Komvopoulos, ${ }^{2}$ and Gabor A. Somorjai ${ }^{1, *}$
}

\begin{abstract}
AUTHORS ADDRESSES:
${ }^{1}$ Department of Chemistry, University of California, Berkeley, California 94720, and

Materials Sciences Division, Lawrence Berkeley National Laboratory, Berkeley, California 94720

${ }^{2}$ Department of Mechanical Engineering, University of California, Berkeley, California 94720
\end{abstract}

AUTHOR EMAIL ADDRESS: somorjai@berkeley.edu

\section{RECEIVED DATE}

TITLE RUNNING HEAD: Pyridine hydrogenation on Pt nanoparticles

\section{CORRESPONDING AUTHOR FOOTNOTE}

*Corresponding author: Tel: 510-642-4053; Fax: 510-643-9668; E-mail: somorjai@ berkeley.edu

${ }^{\ddagger}$ Current address: David H. Koch Institute for Integrative Cancer Research, Massachusetts Institute of Technology, 77 Massachusetts Avenue, Cambridge, MA 02139. 


\begin{abstract}
Pyridine hydrogenation in the presence of a surface monolayer consisting of cubic Pt nanoparticles stabilized by tetradecyltrimethylammonium bromide (TTAB) was investigated by sum frequency generation (SFG) vibrational spectroscopy using total internal reflection (TIR) geometry. TIR-SFG spectra analysis revealed that a pyridinium cation $\left(\mathrm{C}_{5} \mathrm{H}_{5} \mathrm{NH}^{+}\right)$forms during pyridine hydrogenation on the Pt nanoparticle surface, and the $\mathrm{NH}$ group in the $\mathrm{C}_{5} \mathrm{H}_{5} \mathrm{NH}^{+}$cation becomes more hydrogen bound with the increase of the temperature. In addition, the surface coverage of the cation decreases with the increase of the temperature. An important contribution of this study is the in situ identification of reaction intermediates adsorbed on the Pt nanoparticle monolayer during pyridine hydrogenation.
\end{abstract}

KEYWORDS. Heterogeneous catalysis, platinum, nanoparticles, pyridine, hydrogenation, sum frequency generation spectroscopy. 


\section{Introduction}

Basic understanding of catalysis at the molecular level has been the central objective of several recent investigations. ${ }^{1-4}$ Identification of surface intermediates during catalysis is of critical importance in understanding nanoscale catalytic processes. Bridging the materials gap from single crystals to nanoparticles is crucial in elucidating catalysis phenomena encountered in industrially relevant processes. Pyridine $\left(\mathrm{C}_{5} \mathrm{H}_{5} \mathrm{~N}\right)$ is a simple aromatic organic compound structurally related to benzene (one $\mathrm{CH}$ group in the six-member ring is replaced by a nitrogen atom) and is an important building block, solvent, and reagent in organic synthesis. Since the lone pair of electrons at the nitrogen atom is not delocalized into the aromatic $\pi$-system, pyridine is electron donating and exhibits chemical properties similar to those of tertiary amines.

Pyridine is protonated by reaction with acids and forms a positively charged aromatic polyatomic ion known as pyridinium cation $\left(\mathrm{C}_{5} \mathrm{H}_{5} \mathrm{NH}^{+}\right)$. This cation is of particular interest because of its distinct vibrational signature compared to other adsorbates forming during pyridine hydrogenation. ${ }^{5}$ Another desirable effect is that the formation of pyridinium cations is thermodynamically favorable on platinum surfaces. ${ }^{5}$ At a high surface coverage, pyridine adsorbs with the aromatic ring perpendicular to the metal surface through the nitrogen and adjacent carbon atoms in an $\alpha$-pyridyl structure ${ }^{6}$ preventing complete hydrogenation of the ring.

The main objective of this study was to examine surface intermediates during pyridine hydrogenation on monolayers consisting of cubic Pt nanoparticles stabilized by a very thin layer of tetradecyltrimethylammonium bromide (TTAB) under conditions of 10 Torr $\mathrm{C}_{5} \mathrm{H}_{5} \mathrm{~N}$, 100 Torr $\mathrm{H}_{2}$, and 650 Torr $\mathrm{Ar}$ in the temperature range of $300-400 \mathrm{~K}$. This is the first study to provide evidence of a reactive intermediate on a nanoparticle monolayer surface during nanoscale catalysis.

Electron spectroscopy typically cannot be used under ambient pressure conditions of catalytic reactions. Sum frequency generation (SFG) vibrational spectroscopy is a much more effective technique for interface chemical analysis than infrared (IR) absorption and Raman spectroscopy because the SFG signal arises solely from the adsorbates. Materials with centrosymmetry and isotropic gases do not 
appear in the SFG spectrum under the electric dipole approximation. Since bulk Pt has a center of inversion, its contribution to the SFG signal is negligible. Furthermore, a surface specific signal is produced because symmetry is broken at the surface of a Pt crystal. Therefore, SFG spectroscopy and total internal reflection (TIR) geometry were used to study pyridine hydrogenation on a monolayer consisting of Pt nanoparticles in a relatively high temperature range (300-400 K), the main objective being the identification of reactive intermediates during pyridine hydrogenation.

\section{Experimental Procedures}

\subsection{Synthesis and Assembly of Platinum Nanoparticles}

Cubic nanoparticles stabilized with TTAB were prepared by mixing aqueous solutions of $\mathrm{K}_{2} \mathrm{PtCl}_{4}\left(99.9 \%\right.$ pure, Alfa Aesar) and TTAB (99\% pure, Aldrich) in a $20 \mathrm{~mL}$ vial at room temperature. ${ }^{7}$ The mixture was heated to $323 \mathrm{~K}$ for $\sim 5 \mathrm{~min}$ until the solution became clear. After the addition of icecold $\mathrm{NaBH}_{4}\left(98 \%\right.$ pure, Strem Chemicals), the vial was capped with a rubber septum and the $\mathrm{H}_{2}$ gas pressure inside the vial was released through a needle in the septum for $10 \mathrm{~min}$. After the removal of the needle, the solution was kept at $323 \mathrm{~K}$ for $6 \mathrm{~h}$. The cubic nanoparticles were prepared from a solution consisting of $100 \mathrm{mM} \mathrm{TTAB}$ and $1 \mathrm{mM} \mathrm{K}_{2} \mathrm{PtCl}_{4}$ reduced with $30 \mathrm{mM} \mathrm{NaBH}_{4}$. First, the nanoparticles were centrifuged at $3 \mathrm{krpm}$ for $30 \mathrm{~min}$ and the precipitates were discarded. Then, the solution was centrifuged again at $14 \mathrm{krpm}$ for $10 \mathrm{~min}$, the supernatant was discarded, and the precipitates were redispersed in deionized (DI) water by sonication. Finally, the solution was once again centrifuged at 14 $\mathrm{krpm}$ for $10 \mathrm{~min}$, and the precipitates were collected and re-dispersed in DI water. The washed nanoparticles were used in transmission electron microscope (TEM) analysis and reaction studies. The shape and size distributions of the synthesized cubic nanoparticles were studied in a TEM (JEOL 200CX) operated at $200 \mathrm{kV}$.

For spectroscopic measurements, cubic nanoparticles were deposited on a fused quartz prism, using the Langmuir-Blodgett (LB) technique. Colloidal Pt solutions were dispersed on the surface of DI water $(18 \mathrm{M} \Omega \cdot \mathrm{cm})$ sub-phase on a LB trough (type 611, NIMA Technology) at room temperature. The 
surface pressure (monitored with a Wilhelmy plate) was set equal to zero before spreading the nanoparticles. The resulting surface layer was compressed by a mobile barrier at a rate of $20 \mathrm{~cm}^{2} / \mathrm{min}$. The nanoparticles were deposited by lifting the prism that had been immersed in water sub-phase prior to dispersing the nanoparticles at a surface pressure of $\sim 20 \mathrm{mN} / \mathrm{m}$. Representative TEM images of the nanoparticles used in this study have been presented elsewhere. ${ }^{7,8}$ These images show that these cubic Pt nanoparticles do not exhibit any observable tilting with respect to the prism surface.

\subsection{Reaction Cell}

A custom-made cell was used for in-situ SFG spectroscopy in TIR geometry under batch gas circulation. A fused quartz prism coated with a nanoparticle monolayer was pressed against a rubber Oring by tightening a teflon block with two set screws. The temperature at the prism surface was measured with a thermocouple placed directly onto the sample. Heating was applied by wrapping the reactor cell with a heat tape (McMaster-Carr) and applying a variable voltage. Temperature variations across the $20 \times 20 \mathrm{~mm}^{2}$ prism area were insignificant. The reaction cell was connected to a stainless steel tubing gas manifold of diameter equal to $0.3175 \mathrm{~cm}$. Pyridne ( $\geq 99.8 \mathrm{wt} \%$, Fluka) was purified by several freeze-pump-thaw cycles before being introduced into the reaction cell. Before each experiment, pyridine was checked for impurities by gas chromatography (GC). Impurity levels were found to be less than $0.5 \mathrm{wt} \%$ and consisted of mostly light alkanes below $\mathrm{C}_{6}$. A vacuum pressure of less than $1 \mathrm{mTorr}$ was maintained in the reaction cell by mechanical and turbomolecular pumps.

\subsection{Sum Frequency Generation}

A mode-locked Nd:YAG laser with a $1064 \mathrm{~nm}$ fundamental wavelength and a $20 \mathrm{ps}$ pulse width operated at a $20 \mathrm{~Hz}$ repetition rate was used to generate a tunable IR $\left(2750-3600 \mathrm{~cm}^{-1}\right)$ beam and a 532nm second harmonic visible (VIS) beam in the SFG experiments. The IR and VIS beams (25 and $50 \mu \mathrm{J}$, respectively) were spatially and temporally overlapped on the fused quartz surface at incident angles of $55^{\circ}$ and $60^{\circ}$ with respect to the surface normal. SFG-TIR spectra were obtained using ppp (i.e., SFG, VIS, and IR beams all p-polarized) and ssp polarization combinations. The SFG beam was passed 
through a monochromator, and the signal intensity was detected with a photomultiplier tube and a gated integrator while the IR beam was scanned over the frequency range of interest. Because gas molecules absorb some of the incoming radiation, the SFG output was normalized by the intensity of the incident IR beam at the surface. Curve fitting of the SFG-TIR spectra was based on a previously reported procedure, ${ }^{9,10}$ using the following relationship:

$$
I_{S F G} \propto\left|\chi_{N R}^{(2)} e^{i \phi_{N R}}+\sum_{q} \frac{A_{q}}{\omega_{I R}-\omega_{q}+i \Gamma_{q}} e^{i \gamma_{q}}\right|^{2}
$$

where $\chi_{N R}^{(2)}$ is the nonresonant nonlinear susceptibility, $\phi_{N R}$ is the phase associated with the nonresonant background, $A_{q}$ is the strength of the qth vibrational mode, $\omega_{I R}$ is the frequency of the incident IR laser beam, $\omega_{q}$ is the frequency of the qth vibrational mode, $\Gamma_{q}$ is the natural line width of the qth vibrational transition, and $\gamma_{q}$ is the phase associated with the qth vibrational transition. Detailed descriptions of SFG theory can be found elsewhere. ${ }^{11-16}$

\section{Results and Discussion}

Monolayers of TTAB-stabilized Pt nanoparticles were used in the pyridine hydrogenation experiments. The nanoparticles (79\% cubes, $3 \%$ triangles, and $18 \%$ irregular shapes) consisted of only $\operatorname{Pt}(100)$ and were $12.3 \pm 1.4 \mathrm{~nm}$ in size (diagonal). Figure 1 shows SFG spectra (ssp polarization) of a monolayer consisting of TTAB-stabilized cubic Pt nanoparticles obtained before the introduction of pyridine (Figure 1(a)) and during pyridine hydrogenation (10 Torr $\mathrm{C}_{5} \mathrm{H}_{5} \mathrm{~N}, 100$ Torr $\mathrm{H}_{2}$, and 650 Torr Ar) at $300 \mathrm{~K}$ (Figure 1(b)) and $325 \mathrm{~K}$ (Figure 1(c)). Corresponding peak assignments are given in Table 1. The peaks at 2845 and $2935 \mathrm{~cm}^{-1}$ are attributed to symmetric and asymmetric methylene stretches $\left[\mathrm{CH}_{2}(\mathrm{~s})\right.$ and $\mathrm{CH}_{2}(\mathrm{a})$, respectively], the peaks at 2870 and $2955 \mathrm{~cm}^{-1}$ are assigned to symmetric and asymmetric methyl stretches $\left[\mathrm{CH}_{3}(\mathrm{~s})\right.$ and $\mathrm{CH}_{3}(\mathrm{a})$, respectively], while the peak at $2900 \mathrm{~cm}^{-1}$ is attributed to the $\mathrm{CH}_{2}, \mathrm{C}-\mathrm{H}_{\text {distal }}$ stretch. After the introduction of pyridine, an additional peak appeared at $3050 \mathrm{~cm}^{-}$

1. This peak is assigned to the aromatic $-\mathrm{C}=\mathrm{C}-\mathrm{H}$ stretch arising from pyridine adsorbed to the $\mathrm{Pt}$ surface. 
Figure 2 shows SFG spectra ( $p p p$ polarization) of a monolayer consisting of TTAB-stabilized cubic Pt nanoparticles obtained before and after pyridine hydrogenation. The SFG spectrum at the bottom of this figure (acquired before the introduction of pyridine to eliminate the stabilizing agent as a possible source of SFG signal) is featureless in the wavelength range of $3300-3550 \mathrm{~cm}^{-1}$. However, a peak indicative of the pyridinium cation ${ }^{5}$ appeared at $3450 \mathrm{~cm}^{-1}$ after the introduction of pyridine in the presence of hydrogen. The temperature increase resulted in slight red-shifting of the $\mathrm{N}-\mathrm{H}$ peak at $3450 \mathrm{~cm}^{-1}$, possibly indicating an increase in the hydrogen bonding of the NH bonds. This apparent red-shift may also arise from the upcoming $\mathrm{N}-\mathrm{H}$ peak resulting from the temperature rise. As the temperature is increased, the adsorbed pyridine may not be as easily protonated, resulting in the appearance of the $\mathrm{N}-\mathrm{H}$ peak.

Surface coverage $\sigma\left(\right.$ molecules $\left./ \mathrm{cm}^{2}\right)$ by an adsorbate is defined as

$$
\sigma=F \tau
$$

where $F$ is the incident flux (molecules $/ \mathrm{cm}^{2} / \mathrm{s}$ ) and $\tau$ is the residence time given by

$$
\tau=\tau_{0} \exp \left(\frac{\Delta H_{a d s}}{R T}\right)
$$

where $\tau_{0}$ is correlated to the surface atom vibration time (typically $10^{-12} \mathrm{~s}$ ), $\Delta H_{\mathrm{ads}}$ is the heat of adsorption, $T$ is the temperature, and $R$ is the gas constant. According to Eq. (3), surface coverage by an adsorbate decreases exponentially with the increase of the temperature. Hence, the decrease of the peak intensity with the temperature, shown in Figure 2, may be associated with the reduced surface coverage at higher temperatures.

Figure 3 shows ssp and ppp polarized SFG spectra of a monolayer consisting of TTAB-stabilized Pt nanoparticles obtained during pyridine hydrogenation (10 Torr $\mathrm{C}_{5} \mathrm{H}_{5} \mathrm{~N}, 100$ Torr $\mathrm{H}_{2}$, and 650 Torr Ar). In the ssp spectrum (Figure 3(a)), a new peak appears at $3400 \mathrm{~cm}^{-1}$ and is assigned to a $\mathrm{N}-\mathrm{H}$ stretch. ${ }^{17,18}$ The two-fold increase of the signal intensity in the ppp spectrum (Figure 3(b)) suggests that the pyridinium cation is oriented parallel to the surface normal of the prism. It should be noted that this 
does not necessarily imply that the adsorbate is parallel to the nanoparticle surface normal, since the resulting signal may arise from the top of the nanoparticle as well as its sides. For example, if the pyridinium cation were to adsorb in a flat-laying conformation on the nanoparticle sides, the intensity of the $p p p$ spectrum would have been higher than that of the $s s p$ spectrum since the adsorbate would be still parallel to the surface normal of the prism. Earlier studies of pyridine adsorption on Pt single crystals have shown that at a high coverage (>0.6 ML) pyridine adsorbs in an upright conformation, parallel to the surface normal. ${ }^{19-21}$ The pyridinium cation has also been suggested to adopt an upright or tilted conformation, as shown in Figure $4 .^{5}$ Determining the orientation of the adsorbed pyridinium relative to the nanoparticle surface is complex due to the exponential decay of the electric field resulting from the TIR geometry. Consequently, the SFG field may be different on opposite nanoparticle sides, breaking the inversion symmetry. ${ }^{22}$ Assuming that inversion symmetry holds, the top nanoparticle surface will be the dominant contributor to the SFG signal and the pyridinium cation will be oriented in an upright conformation, as shown schematically in Figure 4.

\section{Conclusions}

Pyridine hydrogenation on a monolayer consisting of TTAB-stabilized cubic Pt nanoparticles investigated by TIR-SFG spectroscopy revealed the adsorption of a pyridinium cation intermediate under conditions of 10 Torr $\mathrm{C}_{5} \mathrm{H}_{5} \mathrm{~N}, 100$ Torr $\mathrm{H}_{2}$, and 650 Torr Ar. The $\mathrm{NH}$ group in the pyridinium cation became more hydrogen bound with the increase of the temperature, as suggested by a red-shift in the $\mathrm{N}-\mathrm{H}$ peak, while the decrease in the intensity of this peak at elevated temperatures indicated a decrease in surface coverage of the pyridinium cation. A novel contribution of this study is the in situ identification of reactive intermediates on Pt nanoparticle monolayers during catalytic reactions.

Acknowledgment. This work was supported by the Berkeley-ITRI Research Center (BIRC) under Fund No. 46101-23845-44-EKMAJ, and the Director, Office of Energy Research, Office of Basic Energy Sciences, and Materials Science Division of the U.S. Department of Energy under Contract No. DE- 
AC02-05CH11231. The authors are grateful to S. Habas and Professor P. Yang for nanoparticle monolayer synthesis.

\section{References and Notes}

(1) Somorjai, G. A. Journal of Molecular Catalysis A: Chemical 1996, 107, 39-53.

(2) Zaera, F. Progress in Surface Science 2001, 69, 1-98.

(3) LeBlanc, R. J.; Chu, W.; Williams, C. T. Journal of Molecular Catalysis A: Chemical 2004, 212, 277-289.

(4) Williams, C. T.; Yang, Y.; Bain, C. D. Langmuir 2000, 16, 2343-2350.

(5) Lee, I. C.; Masel, R. I. Journal of Physical Chemistry B 2002, 106, 368-373.

(6) Connolly, M.; Somers, J.; Bridge, M. E.; Lloyd, D. R. Surface Science 1987, 185, 559568.

(7) Lee, H.; Habas, S. E.; Kweskin, S. J.; Butcher, D.; Somorjai, G. A.; Yang, P. Angewandte Chemie-International Edition 2006, 45, 7824-7828.

(8) Bratlie, K. M.; Lee, H.; Komvopoulos, K.; Yang, P.; Somorjai, G. A. Nano Letters 2007, 7, 3097-3101.

(9) Bain, C. D.; Davies, P. B.; Ong, T. H.; Ward, R. N.; Brown, M. A. Langmuir 1991, 7, 1563-1566.

(10) Moore, F. G.; Becraft, K. A.; Richmond, G. L. Applied Spectroscopy 2002, 56, $1575-$ 1578.

(11) Kung, K. Y.; Chen, P.; Wei, F.; Rupprechter, G.; Shen, Y. R.; Somorjai, G. A. Review of Scientific Instruments 2001, 72, 1806-1809.

(12) Yang, M.; Tang, D. C.; Somorjai, G. A. Review of Scientific Instruments 2003, 74, 45544557.

(13) Bratlie, K. M.; Flores, L. D.; Somorjai, G. A. Surface Science 2005, 599, 93-106.

(14) Shen, Y. R. The Principles of Nonlinear Optics; Wiley: New York, 2003. 
(15) Shen, Y. R. Annual Review of Physical Chemistry 1989, 40, 327-350.

(16) Shen, Y. R. Nature 1989, 337, 519-525.

(17) Kang, D. H.; Trenary, M. Surface Science 2000, 470, L13-L19.

(18) Kang, D. H.; Trenary, M. Surface Science 2002, 519, 40-56.

(19) Horsley, J. A.; Stohr, J.; Hitchcock, A. P.; Newbury, D. C.; Johnson, A. L.; Sette, F. Journal of Chemical Physics 1985, 83, 6099-6107.

(20) Haq, S.; King, D. A. Journal of Physical Chemistry 1996, 100, 16957-16965.

(21) Grassian, V. H.; Muetterties, E. L. Journal of Physical Chemistry 1987, 91, 389-396.

(22) Yeganeh, M. S.; Dougal, S. M.; Silbernagel, B. G. Langmuir 2006, 22, 637-641. 


\section{List of Tables and Figures}

Table 1. Peak assignments in SFG spectra of a monolayer consisting of TTAB-stabilized cubic Pt nanoparticles obtained before and during pyridine hydrogenation (10 Torr $\mathrm{C}_{5} \mathrm{H}_{5} \mathrm{~N}, 100$ Torr $\mathrm{H}_{2}$, 650 Torr Ar, and temperature in the range of $300-400 \mathrm{~K}$ ).

Figure 1. SFG spectra ( $s p$ polarization) of a monolayer consisting of TTAB-stabilized cubic Pt nanoparticles obtained (a) before and (b),(c) during pyridine hydrogenation $\left(10\right.$ Torr $\mathrm{C}_{5} \mathrm{H}_{5} \mathrm{~N}, 100$ Torr $\mathrm{H}_{2}$, and 650 Torr Ar) at (b) $300 \mathrm{~K}$ and (c) $325 \mathrm{~K}$. Markers represent experimental data and solid lines curve fits that follow Eq. (1).

Figure 2. SFG spectra ( $p p p$ polarization) of a monolayer consisting of TTAB-stabilized cubic Pt nanoparticles showing the pyridinium $\mathrm{N}-\mathrm{H}^{+}$band before (bottom spectrum) and during pyridine hydrogenation (10 Torr $\mathrm{C}_{5} \mathrm{H}_{5} \mathrm{~N}, 100$ Torr $\mathrm{H}_{2}$, 650 Torr Ar, and temperature in the range of 300-400 K). Markers represent experimental data and solid lines curve fits that follow Eq. (1).

Figure 3. SFG spectra of a monolayer consisting of TTAB-stabilized cubic Pt nanoparticles for (a) ssp and (b) ppp polarization. The spectra show the pyridinium $\mathrm{N}-\mathrm{H}^{+}$and $\mathrm{N}-\mathrm{H}$ bands during pyridine hydrogenation (10 Torr $\mathrm{C}_{5} \mathrm{H}_{5} \mathrm{~N}, 100$ Torr $\mathrm{H}_{2}, 650$ Torr Ar, and temperature of $300 \mathrm{~K}$ ). Markers represent experimental data and solid lines curve fits that follow Eq. (1).

Figure 4. Schematic diagram of two possible configurations of the pyridinium cation $\left(\mathrm{C}_{5} \mathrm{H}_{5} \mathrm{NH}^{+}\right)$ intermediate. The green, gray, and blue spheres represent carbon, hydrogen, and nitrogen atoms, respectively. 
Table 1. Peak assignments in SFG spectra obtained from a monolayer consisting of TTAB-stabilized cubic Pt nanoparticles before and after pyridine hydrogenation under conditions of 10 Torr $\mathrm{C}_{5} \mathrm{H}_{5} \mathrm{~N}, 100$ Torr $\mathrm{H}_{2}, 650$ Torr Ar, and temperature in the range of 300-400 K.

\begin{tabular}{cc}
\hline Wavenumber $\left(\mathrm{cm}^{-1}\right)$ & Peak assignment (surface) \\
\hline 3450 & $\mathrm{~N}-\mathrm{H}^{+}$(pyridine) \\
3400 & $\mathrm{~N}-\mathrm{H}$ (pyridine) \\
3050 & $-\mathrm{C}=\mathrm{C}-\mathrm{H}$ (pyridine) \\
2955 & $\mathrm{CH}_{3}(\mathrm{a})$ (TTAB) \\
2935 & $\mathrm{CH}_{2}(\mathrm{a})$ (TTAB) \\
2900 & $\mathrm{CH}_{2}, \mathrm{C}-\mathrm{H}_{\text {distal }}(\mathrm{TTAB})$ \\
2870 & $\mathrm{CH}_{3}(\mathrm{~s})$ (TTAB) \\
2845 & $\mathrm{CH}_{2}$ (s) (TTAB) \\
\hline \hline
\end{tabular}






Figure 1 




Figure 2 


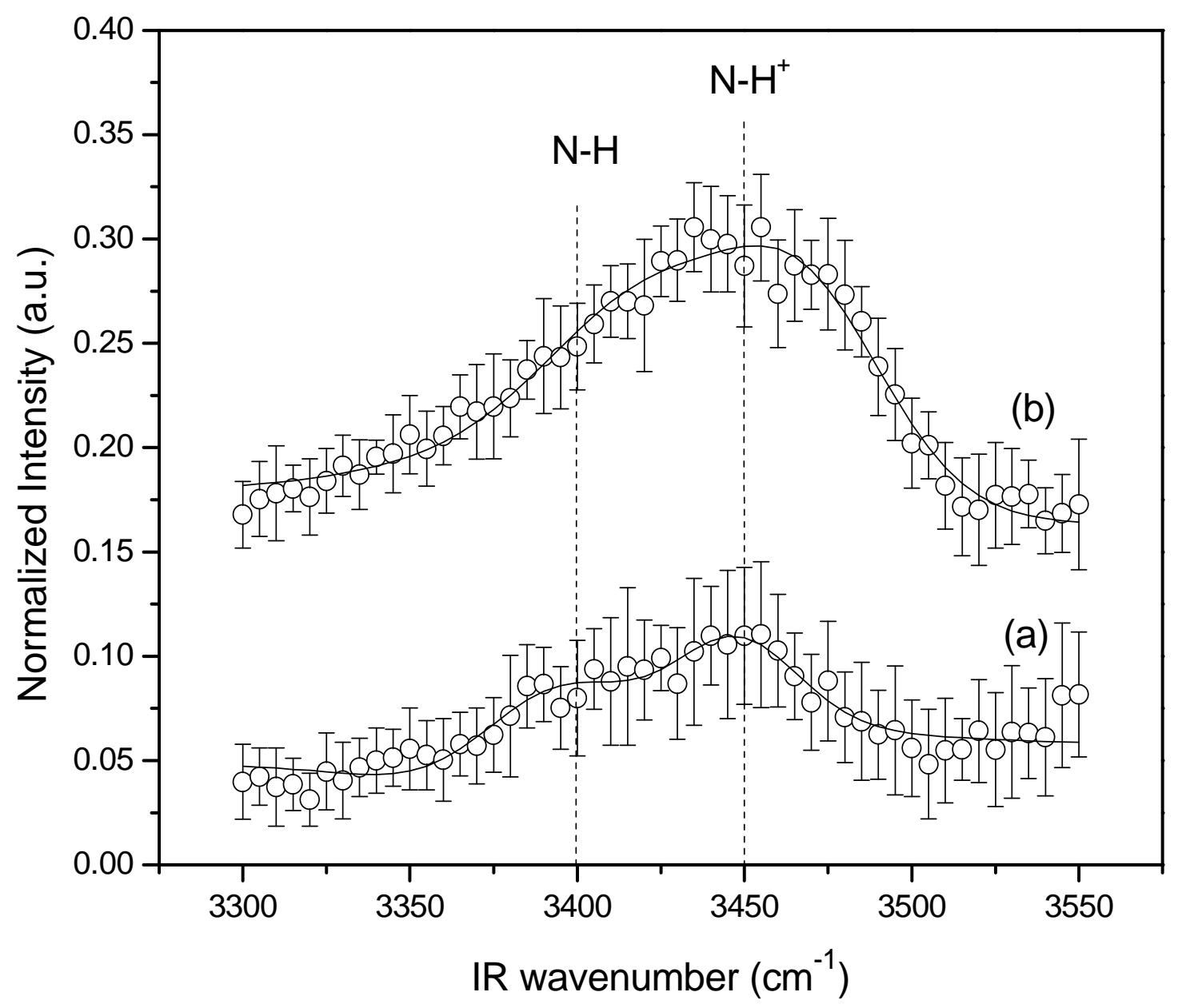

Figure 3 

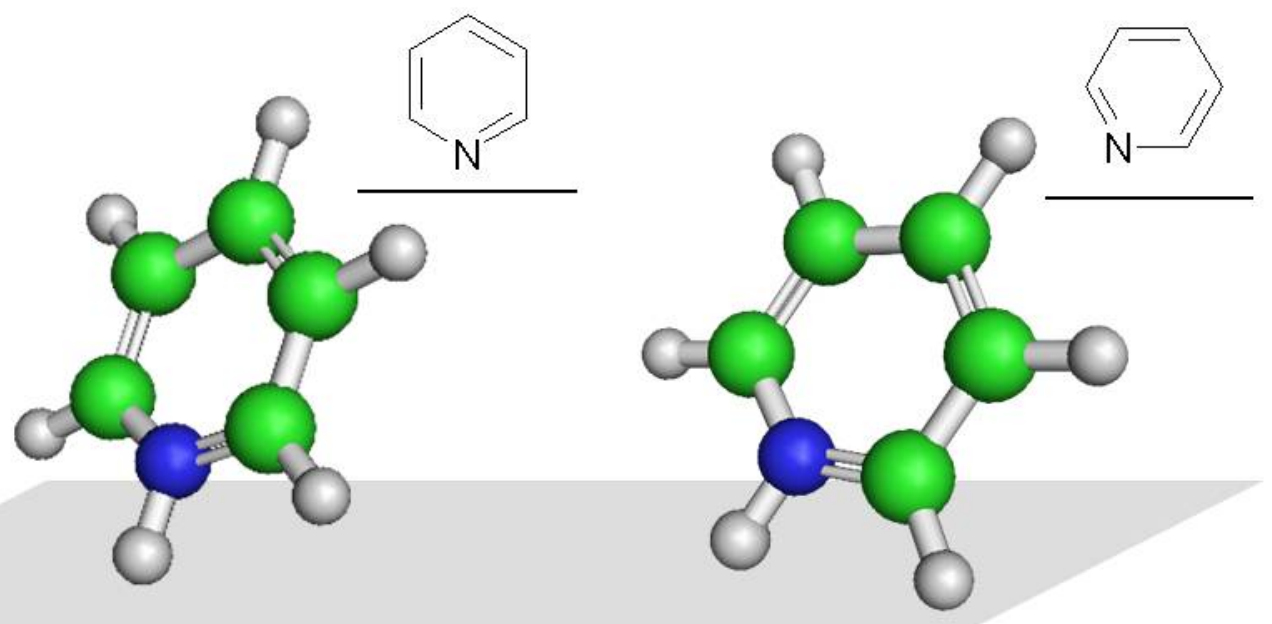

Figure 4 